\title{
Early Changes in Parathyroid Hormone Response and Proteoglycan Synthesis of Chick Embryonic Femur Produced by Exposure to 6-Aminonicotinamide in ovo
}

\author{
Kohtaro KAWASHIMA, Sei-IChi TANUMA and Hiroyoshi ENDO \\ Department of Physiological Chemistry, Faculty of Pharmaceutical Sciences, \\ Teikyo University, Sagamiko, Kanagawa, 199-01, Japan
}

\begin{abstract}
The mechanism of induction of micromelia in 6-day-old chick embryo by 6-aminonicotinamide (6-AN) was investigated. Six-day-old chick embryo exposed to 6-AN did not show micromelia when tenfold excess of nicotinamide over 6-AN was co-administered. The ability of nicotinamide to prevent the induction of micromelia was partially offset after $4 \mathrm{hr}$ of exposure to 6-AN and completely disappeared after $6 \mathrm{hr}$. The length of time necessary for the induction of micromelia was not affected by the concentration of 6-AN. These results indicate that exposure to 6-AN for only a short period of $6 \mathrm{hr}$ is sufficient to commit the limb to micromelia and that cellular components involved in the induction of micromelia alter during this period. During this period, newly synthesized proteoglycan monomers typical of cartilage decreased in average molecular size, and isolated femora did not respond to parathyroid hormone (PTH) but to dibutyryl cyclic AMP to stimulate growth of cartilage in organ culture.
\end{abstract}

A nicotinamide analogue, 6-aminonicotinamide (6-AN) is known to affect $\operatorname{limb}$ development preferentially, thereby inducing micromelia (Landauer, W., 1957; Caplan, A. I., 1971 ; Caplan, A. I., 1972a, b ; Seegmiller, R. E., et al., 1972; Schubert, D., and Lacorbiere, M., 1976 ; Seegmiller, R. E., 1977 ; Mclachlan, J. C., 1980). Although the precise mechanism has not yet been elucidated, it has been reported that 6-AN acts by suppressing $\mathrm{NAD}(\mathrm{P})$-dependent reactions (Landauer, W., 1957 ; Dietrich, L. S., et al., 1958 ; Verrusio, A. C., et al., 1968; Ritter, E. J., et al., 1975; Sheffeils, V. C., and

Received August 1, 1986
Seegmiller, R. E., 1980) and sulfate utilization to form sulfated proteoglycans (Caplan, A. I., 1972a, b; Seegmiller, R. E., et al., 1972; Overman, D. O., et al., 1972; Honda, A., et al., 1982), and by changing the developmental pathways of mesodermal cells (Caplan, A. I., 1972a, b; Schubert, D., and Lacorbiere, M., 1976)

As a first step toward elucidating the mechanism, it is important to determine the exposure time of chick embryo to 6-AN required to induce micromelia. The effect of 6-AN was completely offset by the co-administration of nicotinamide (Landauer, W., 1957 ; Caplan, A. I., 1971 ; Caplan, A. I., 1972a). To determine the shortest exposure 
time required to induce micromelia with 6$\mathrm{AN}$, we administered nicotinamide to chick embryos at varying times after exposure to 6-AN. We found that no alleviating effect was observed when nicotinamide was introduced any time after $6 \mathrm{hr}$ of exposure to 6 - AN, indicating that at least $6 \mathrm{hr}$ of exposure to 6-AN was sufficient to commit limbs to micromelia. During this short period, 6-AN causes cellular alterations that are resistant to the administration of nicotinamide.

To examine the cellular alteration which occurred during this period, it may be instructive to consider alterations in hormone actions which affect growth of cartilage and synthesis of cartilage-specific macromolecules. We showed previously that PTH stimulated the growth of embryonic chick limbs in organ culture (Kawashima, K., et al., 1980a, b) but not the growth of micromelic limbs exposed to 6-AN for 5 days in ovo (Kawashima, K., et al., 1982). In this paper, we examined whether exposure to $6-\mathrm{AN}$ for $6 \mathrm{hr}$ in ovo leads to a change in the response of limbs to PTH in vitro. We provide evidence that on short exposure $(6 \mathrm{hr})$, the limbs lose their response to PTH. Moreover, we show that the average molecular size of newly synthesized proteoglycan monomers characteristic of cartilage decreases during this short term treatment.

\section{Experimental Procedures}

\section{Materials}

6-Aminonicotinamide and dibutyryl cyclic AMP were purchased from Sigma Chemical Co. Nicotinamide was obtained from Wako Pure Chemical Industries Ltd., Japan. Parathyroid Hormone (Bovine, 1-34) was purchased from Beckman Instruments Inc.

\section{Treatment of agents in ovo}

A window was made in White Leghorn eggs after incubation at $37^{\circ} \mathrm{C}$ and $60 \%$ relative humidity for 6 days (stage 28-29) (Hamberger, V., and Hamilton, H. L., 1951) and various concen- trations of 6-AN or nicotinamide in $50 \mu \mathrm{l}$ of $\mathrm{Mg}^{2+}, \mathrm{Ca}^{2+}$-free phosphate buffered saline (PBS(-)) were introduced. The same volume of PBS(-) was introduced into control eggs.

\section{Isolation and cultivation of femur}

Isolation of femora from chick embryos was performed as described previously (Kawashima, K., et al., 1980a, b). The isolated femora were clutivated in a roller tube (Endo, H., 1960) in a chemically defined medium BGJb-HW2 (Endo, H., 1974). Pair-mate cultures were employed: one femur from the embryo served as a control and the other was cultivated in medium with PTH or dibutyryl cyclic AMP.

\section{Analysis of proteoglycan}

Extraction and characterization of proteoglycans were carried out essentially as described by Hascall and Stanley (Hascall, V. C., and Stanley, W. S., 1969). ${ }^{35}$ S-Labeled proteoglycans were extracted from isolated femora at $4^{\circ} \mathrm{C}$ in $4 \mathrm{M}$ guanidine- $\mathrm{HCl}, 50 \mathrm{mM}$ sodium acetate $(\mathrm{pH} 5.8)$ containing $1 \mathrm{mM}$ phenylmethylsulfonylfluoride for $24 \mathrm{hr}$. Solid $\mathrm{CsCl}$ was added to the extracts to give a density of $1.50 \mathrm{~g} / \mathrm{ml}$ and a density gradient was formed by sedimentation at 40,000 r.p.m. for $44 \mathrm{hr}$ at $22^{\circ} \mathrm{C}$. The bottom fractions $(\rho=1.53)$ were pooled. Samples were dialyzed against the extraction solution and condensed by Sehadex G100 . The proteoglycan solution in $4 \mathrm{M}$ guanidine$\mathrm{HCl}$ containing $50 \mathrm{mM}$ sodium acetate $(\mathrm{pH} 5.8)$ was layered on a linear $5-20 \%$ sucrose gradient in $4 \mathrm{M}$ guanidine- $\mathrm{HCl}$ containing $50 \mathrm{mM}$ sodium acetate ( $\mathrm{pH}$ 5.8). The sedimentation was carried out at 38,000 r.p.m. for $14 \mathrm{hr}$ at $4^{\circ} \mathrm{C}$ (Kimata, et al., 1974).

\section{Results}

Effect of nicotinamide on induction of micromelia by 6-AN

To confirm the exsistence of the counteraction of nicotinamide to the teratogenic action of 6-AN, we examined the dose-dependency of the effect on the length of embryonic chick femora.

As shown in Fig. 1, the length resumed exponentially with the increase in the concentration of nicotinamide up to $50 \mu \mathrm{g} / \mathrm{egg}$. 
Higher concentrations than $100 \mu \mathrm{g} / \mathrm{egg}$ resulted in complete resumption. Accordingly, a tenfold excess of nicotinamide over 6-AN was used in subsequent experiments.
Time necessary for induction of micromelia by 6- $A N$

If the effect of 6-AN is completed in a specific period, the administration of nicotin-

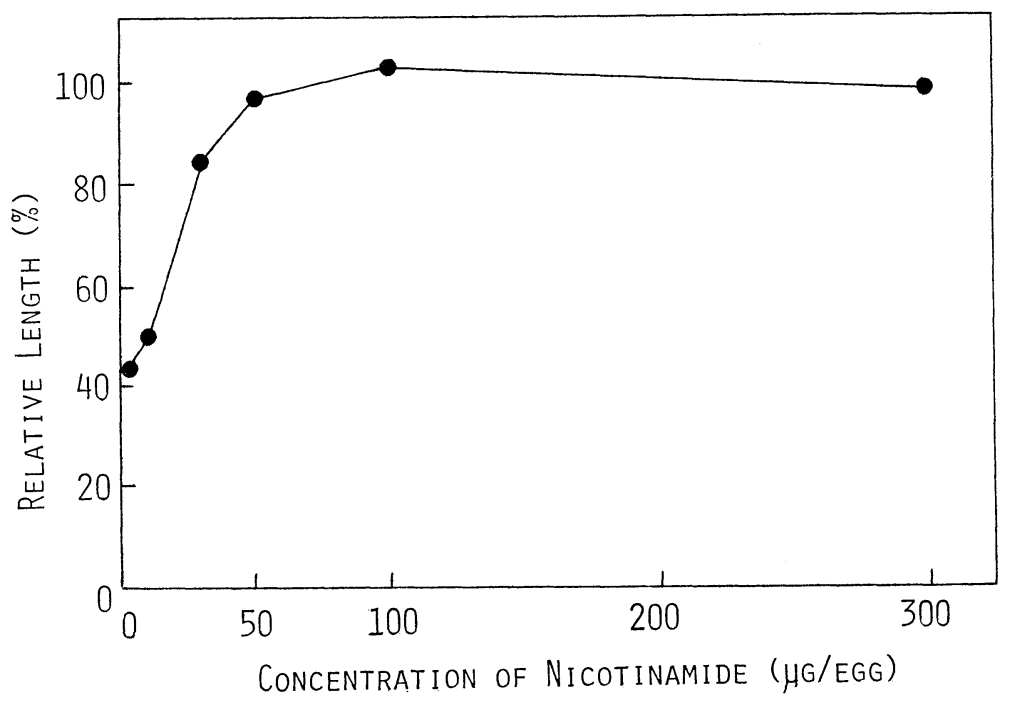

Fig. 1. Dose-dependent effect of co-administration of nicotinamide and 6-AN on induction of micromelia. The concentrations of nicotinamide indicated were administered with 6AN $(10 \mu \mathrm{g} / \mathrm{egg})$ to 6 -dayold chick embryos. The eggs were incubated for 3 day and then the femora were isolated and their length was measured. Length were shown as percentages for the length of control femora (5.90 \pm $0.24 \mathrm{~mm}$ ). Points are means for five samples and standard deviation was so small that its representation did not exceed the diameter of each point.

Fig. 2. Effect of administration of nicotinamide after exposure to 6-AN on induction of micromelia. Varying concentrations of 6-AN $(5(\boldsymbol{\Delta}), 10(\mathbf{O}), 20(\mathbf{\square}) \mu \mathrm{g} / \mathrm{egg})$ were administered to 6-day-old chick embryos. Nicotinamide $(100 \mu \mathrm{g} / \mathrm{egg})$ was administered at the indicated times after exposure to 6-AN. After further incubation of the embryos for 3 days, the femora were isolated and their length was measured. Values are shown as percentages of the length of control femora $(6.03 \pm 0.29)$. The length of femora of embryos that were not treated with nicotinamide is shown by open symbols. Points are means for five samples and standard deviation was so small that its representation is buried in each symbol.

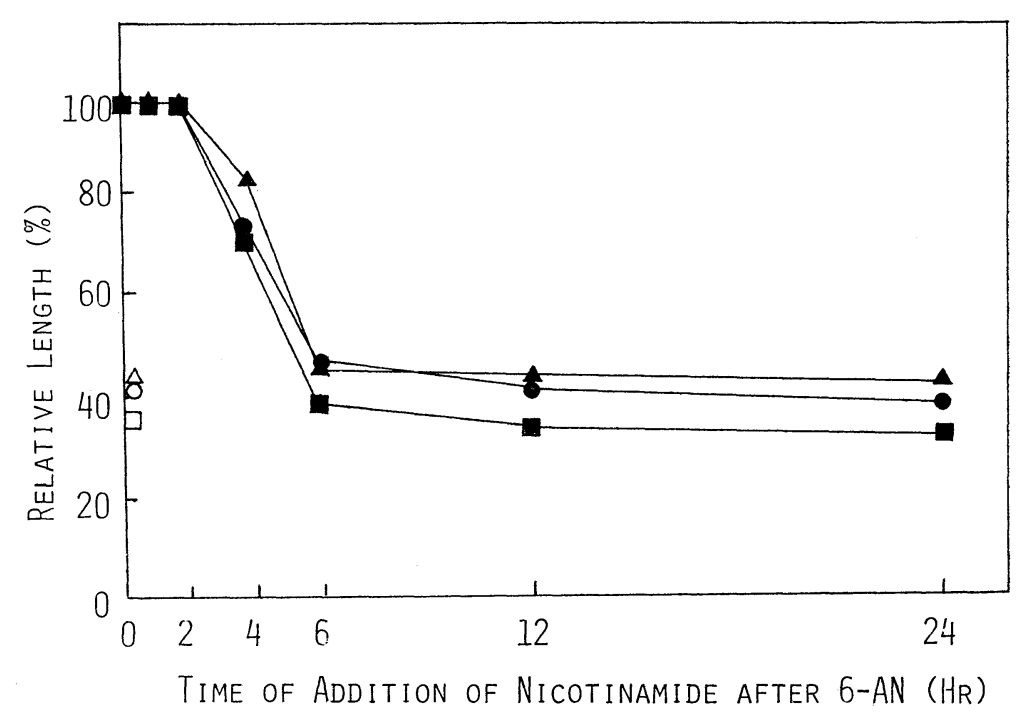


amide after this period should not modify the effect of 6-AN at all.

Nicotinamide alleviated the effect of 6AN when it was administered within $2 \mathrm{hr}$ after exposure to 6-AN (Fig. 2). However, its effect in preventing micromelia gradually decreased thereafter, and it had no effect after exposure to $6-\mathrm{AN}$ for $6 \mathrm{hr}$ or more. We also examined the relationship between the concentration of $6-\mathrm{AN}$ and the time necessary for the induction of micromelia. The results showed that the length of exposure required to induce micromelia was identical irrespective of the concentration of 6-AN used (5, 10 and $20 \mu \mathrm{g} / \mathrm{egg}$ ) (Fig. 2).

\section{Effect of 6-AN on proteoglycan population}

Fig. 3 shows sedimentation profiles in sucrose gradient of sulfated proteoglycans synthesized by femora exposed to 6-AN for $6 \mathrm{hr}$ as compared to those by control femora. The proteoglycan monomers synthesized by control femora were separated into two size classes on the gradient. The heavy proteoglycan (fraction 1-12) is synthesized specifically by chondrocytes and the light proteoglycan (fraction 20-24) is synthesized by fibroblasts, blastodisc and spinal cord cells (Okayama, M., et al., 1976). The figure shows that slowly migrated heavy proteoglycan monomers increased in 6-AN-exposed femora. The figure also shows that the overall proportion of heavy to light sulfated proteoglycans in 6-AN-exposed femora is similar to that in control femora.

Effect of 6-AN on the response of limbs to PTH

Embryos were treated in ovo in three different ways, as described in Fig. 4, and then the femora were isolated and cultivated in BGJb-HW2 containing PTH or dibutyryl cyclic AMP and measured their length. Both PTH and dibutyryl cyclic AMP stimulated growth of control femora (Fig. 4A). In contrast, PTH had no stimulatory effect on femora isolated from embryos that had been exposed continuously to 6-AN (Fig. 4B). The loss of response to PTH was also fixed in femora that had been exposed to 6-AN for $6 \mathrm{hr}$ before administration of nicotinamide (Fig. 4C). In femora exposed to

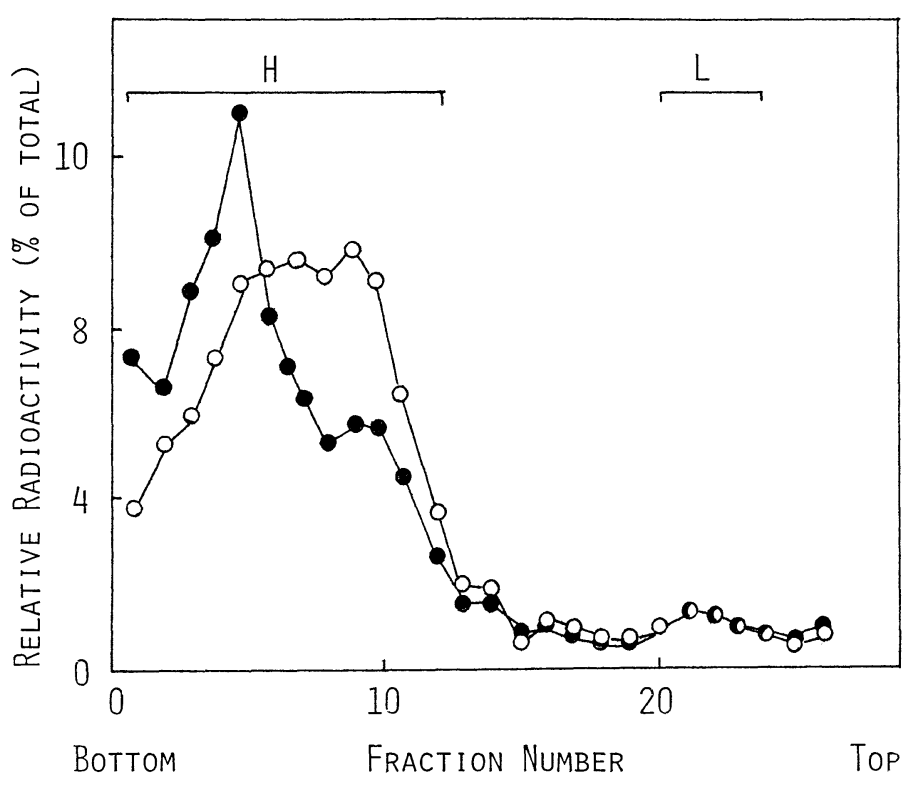

Fig. 3. Sedimentation profiles in sucrose gradient of proteoglycans from control (O) and 6-AN $(10 \mu \mathrm{g} / \mathrm{egg})$ exposed $(\bigcirc)$ femora. Six-day-old chick embryos were labeled with $\left({ }^{35} \mathrm{~S}\right)$ sulfate $(10 \mu \mathrm{Ci} /$ egg) for $2 \mathrm{hr}$ at $37^{\circ} \mathrm{C}$ after exposure to 6-AN for $6 \mathrm{hr}$. ${ }^{35} \mathrm{~S}$-labeled proteoglycans were extracted from isolated femora and analyzed by sedimentation of sucrose gradient as described under Experimental Procedures. Fractions 1-12 and 20-24 were collected as heavy (H) and light (L) proteoglycans respectively. 
6-AN with or without subsequent administration of nicotinamide (Fig. 4B and 4C), however, growth stimulation by dibutyryl cyclic AMP was observed as in the case of control femora (Fig. 4A).

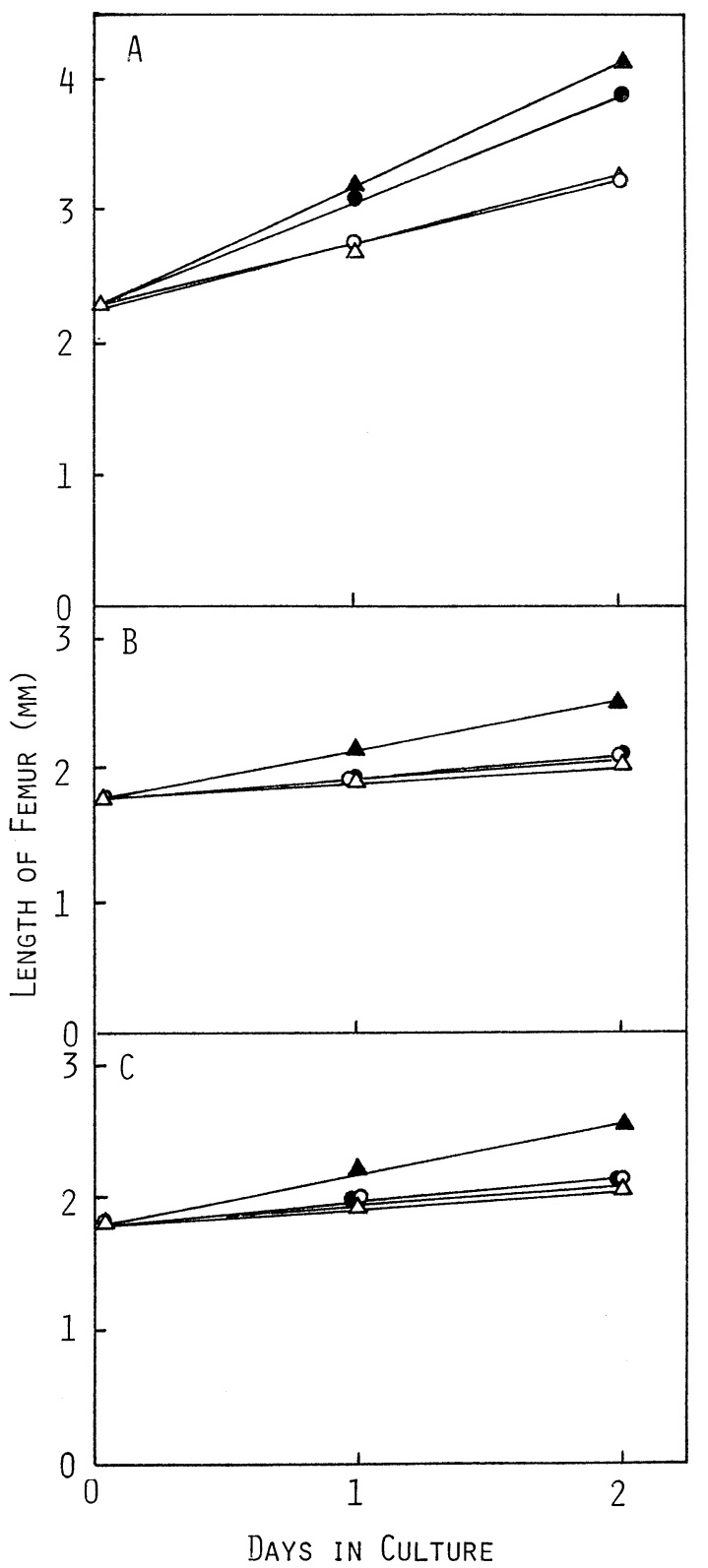

\section{Discussion}

We have investigated the mechanism of induction of micromelia by 6-AN. This report focused on how long 6-AN had to act to induce micromelia and what early events are involved in the induction of micromelia.

Exposure to 6-AN for $6 \mathrm{hr}$ was enough for the completion of micromelia and the length of exposure time to induce micromelia was not affected by the concentration of 6-AN (Fig. 2). These results indicate that changes in cellular components are necessary to induce micromelia. We showed previously (Kawashima, K., et al., 1980a, b) that growth of isolated embryonic chick femora cultivated in a chemically defined medium BGJb-HW2 was stimulated by PTH or diblutyryl cyclic AMP. PTH response in stimulating the growth of limb, however, was completely disappeared by the long exposure (from day 4 to day 9) of 6-AN in ovo (Kawashima, K., et al., 1982). In addition, proteoglycan synthesis was altered by long term exposure to 6-AN. Seegmiller,

Fig. 4. Effect of PTH and dibutyryl cyclic AMP on growth of femora treated in three different ways. (A) Femora were isolated from 7-dayold chick embryos (control). (B) Femora were isolated from 7-day-old chick embryos that had been exposed to 6-AN (10 $\mu \mathrm{g} / \mathrm{egg})$ for $30 \mathrm{hr}$. (C) Femora were isolated from 7-dayold chick embryos that had been exposed to 6-AN $(10 \mu \mathrm{g} / \mathrm{egg})$ for $6 \mathrm{hr}$ and treated with nicotinamide $(100 \mu \mathrm{g} / \mathrm{egg})$ and then incubated for $24 \mathrm{hr}$. These isolated femora were cultivated in BGJb-HW2 with $(\mathbf{O})$ or without $(\bigcirc)$ PTH $(5 \mathrm{U} / \mathrm{ml})$ and with $(\Delta)$ or without $(\Delta)$ $1 \mathrm{mM}$ dibutyryl cyclic AMP. The length of the femora was measured every day. Points are means for five replicate cultures. The standard error was so small that its representation did not exceed the diameter of each point. 
et al. (Seegmiller, R. E., et al., 1980) demonstrated that when cartilage epiphyses isolated from 11-day-old chick embryos were cultivated in vitro in the presence of 6-AN for $17 \mathrm{hrs}$, the molecular weight of chondroitin sulfate and the ratio of the amount of chondroitin-4-sulfate to 6-sulfate were altered. Honda et al demonstrated that three day's exposure of 6-AN to chick embryo in ovo (day 4 to day 7) resulted in the inhibition of total glycosaminoglycan synthesis and of transition of mesenchymal to cartilage specific proteoglycans with an increase in the ratio of low sulfated glycosaminoglycans (Honda, A., et al., 1982). Exposure of 6AN to 10-day-old chick embryonic limb for one day in vitro also impaired the glycosaminoglycan synthesis (Iwama, M., et al., 1983). We therefore investigated whether PTH response and the structure of sulfated proteoglycans of the femora were altered by short time exposure $(6 \mathrm{hr})$ to $6-\mathrm{AN}$ in ovo. Fig. 4 shows that after even short exposure to $6-\mathrm{AN}(6 \mathrm{hr})$ the limbs lose their response to PTH. Moreover, during this period, proteoglycan monomers typical of cartilage decreased in average molecular size (Fig. 3). As previously mentioned, PTH stimulated the synthesis of proteoglycan in chondrocytes in cell culture (Suzuki, F., et al., 1976) and in chick embryonic limb in organ culture (Kawashima, K., et al., 1980a, b). These observations, therefore, imply that exposure to 6-AN would primarily cause changes in the response of chondrocytes to PTH and secondarily changes in the metabolism of chondrocyte-specific proteoglycan. It is possible that the changes resulting in micromelia involve the absence of PTH dependent cartilage growth and differentiation. The altered extracellular macromolecules such as proteoglycan and collagen may be involved in the maintenance of micromelia.

Poly (ADP-ribose) is a variably sized homopolymer synthesized from NAD and modifies chromosomal protein. Cherney et al. (Cherney, B. W., et al., 1985) showed that poly (ADP-ribose) synthetase, which catalyzes the synthesis of poly (ADP-ribose), transiently increased when chick embryonic mesenchymal cells differentiate into muscle and cartilage. Therefore, modification of chromosomal proteins by poly (ADP-ribose) may be one of the important events in normal development of cartilage. We found the inhibition of poly (ADP-ribose) synthetase activity and the changes in de novo synthesis of chromosomal proteins in the femora exposed to 6-AN for $6 \mathrm{hr}$ in ovo (data not shown). Since changes in de novo synthesis and modification of chromosomal proteins occur during an extensive reprogramming of genetic expression, it seems possible that exposure to 6 -AN causes changes in the transcription in chondrocytes and results in loss of responsiveness to $\mathrm{PTH}$, probably involving deterioration of PTH receptor. It is felt that suppression of gene expression of PTH receptor would be an early event in inducing micromelia.

Another possible mechanism inducing micromelia by 6-AN is disturbance of $\mathrm{NAD}(\mathrm{P})$-dependent enzyme reaction. 6-AN markedly lowers the level of ATP in limb bud (Sheffield, V. C., and Seegmiller, R. E., 1980 ; Honda, A. et al., 1982). These studies suggest that 6-AN forms an abnormal NAD analogue which inhibits the activity of NAD $(P)$-dependent enzymes associated with the production of ATP. 6-AN acts preferentially on $\operatorname{limb}$ to induce micromelia. However, it is not known whether this impairment of the NAD(P)-dependent enzyme reaction occurred specifically in limb or not. Comparative studies on $\operatorname{limb}$ and the other tissues are necessary. Chondrocytes, however, require a large amount of ATP for the formation of $3^{\prime}$-phosphoadenosine $5^{\prime}$ phosphosulfate (PAPS) which is essential for the synthesis of sulfated proteoglycans, a major component of cartilage matrix. The decrease in ATP content, therefore, may be more sensitive to inhibition of differentiation and proliferation of chondrocytes than of 
other cells, thereby inducing micromelia.

Prostaglandin $\mathrm{E}_{1}$ and $\mathrm{E}_{2}$ also stimulate in vitro chondrogenic differentiation of mesodermal cell of embryonic chick limb bud by increasing of cAMP (Kosher, R. T., and Walker, K. H., 1983) and play an essential role in limb development (Gay, S. W., and Kosher, R. A., 1985; Chepenik, K. P., et al., 1984). Therefore the effects of $\mathrm{PGE}_{1}$ and $\mathrm{PGE}_{2}$ on the growth and differentiation of limbs of micromelia are an interesting subject. Experiments on this are in progress.

The present studies should serve as a basis for more detailed investigations on not only the mechanism of induction of micromelia by 6-AN but also the biological significance of PTH and extracellular macromolecules in limb development and differentiation.

\section{References}

Caplan, A. I. (1971). The teratogenic action of the nicotinamide analogues 3-acetylpyridine and 6-aminonicotinamide on developing chick embryos. J. Exp. Zool. 178, 351-358.

Caplan, A. I. The site and sequence of action of 6-aminonicotinamide in causing bone malformations of embryonic chick limb and its relationship to normal development. (1972a) Dev. Biol. 28, 71-83.

Caplan, A. I. (1972b). Effect of a nicotinamidesensitive teratogen 6-aminonicotinamide on chick limb cells in culture. Exp. Cell Res. 70, 185.

Chepenik, K. P., W. C. Ho, B. M. M. Waite and C. L. Parker (1984). Arachidonate metabolism during chondrogenesis in vitro. Calcif. Tissue Int. 36, 175-181.

Cherney, B. W., R. J. Midura and A. I. Caplan (1985). Poly (ADP-ribose) synthetase and chick limb mesenchymal cell differentiation. Dev. Biol. 112, 115-125.

Dietrich, L. S., I. M. Friedland and L. A. Kaplan (1958). Pyridine nucleotide metabolism: Mechanism of action of the niacin antagonist, 6aminonicotinamide. J. Biol. Chem. 233, 964-968.

Endo, H. (1974). Cartilage matrix metabolism of chondrocytes changing through their life span and its possible hormonal control, as demonstrated by tissue culture studies. (In Japanese) Connective Tissue 6, 139-148.

Endo, H. (1960). Ossification in tissue culture I. Histological development of the femur of chick embryo in various liquid media. Exp. Cell Res. 21, 151-163.

Gay, S. W. and R. A. Kosher (1985). Prostaglandin synthesis during the course of limb cartilage differentiation in vitro. J. Embryol. Exp. Morph. 89, 367-382.

Hamberger, V. and H. L. Hamilton (1951). A series of normal stages in the development of the chick embryo. J. Morph. 88, 49-92.

Hascall, V. C. and W. S. Stanley (1969). Proteinpolysaccharide complex from bovine nasal cartilage: The function of glycoprotein in the formation of aggregates. J. Biol. Chem. 244, 2384-2396.

Honda, A., M. Iwama, T. Umeda and Y. Mori (1982). The teratogenic mechanism of 6-aminonicotinamide on limb formation of chick embryos: Abnormalities in the biosynthesis of glycosaminoglycans and proteoglycans in micromelia. J. Biochem. (Tokyo) 91, 1959-1970.

Iwama, M., A. Honda and Y. Mori (1983). Alterations of glycosaminoglycans synthesized by chick embryo cartilage treated with 6-aminonicotinamide J. Pharmacobiodyn. 6, 613-620.

Kawashima, K., S. Iwata and H. Endo (1980a). Growth stimulatory effect of parathyroid hormone, calcitonin and $\mathrm{N}^{6}, \mathrm{O}^{2 \prime}$-dibutyryl adenosine $3^{\prime} ; 5^{\prime}$-cyclic monophosphoric acid on chick embryonic cartilage cultivated in a chemically defined medium. Endocrinol. Japon. 27, 349-356.

Kawashima, K., S. Iwata and H. Endo (1980b). Selective activation of diaphyseal chondrocytes by parathyroid hormone, calcitonin and $\mathrm{N}^{6}, \mathrm{O}^{2}$ dibutyryl adenosine $3^{\prime} ; 5^{\prime}$-cyclic monophosphoric acid in proteoglycan synthesis in vitro. Endocrinol. Japon. 27, 357-361.

Kawashima, K., S. Tanuma and H. Endo (1982). Absence of response of chick embryonic limb to the growth stimulatory effect of parathyroid hormone in vitro after exposure to 6-aminonicotinamide in ovo. Endocrinol. Japon. 29, 191-195.

Kimata, K., M. Okayama and S. Suzuki (1974). Heterogeneity of proteochondroitin sulfates produced by chondrocytes at different stages of cytodifferentiation. J. Biol. Chem. 249, 1646-1653. 
Kosher, R. T. and K. H. Walker (1983). The effect of prostaglandins on in vitro limb cartilage differentiation. Exp. Cell Res. 145, 145153.

Landauer, W. (1957). Niacin antagonists and chick development. J. Exp. Zool. 136, 509530.

Mclachlan, J. C. (1980). The effect of 6-aminonicotinamide on limb development. J. Embryol. Exp. Morph. 55, 307-318.

Okayama, M., M. Pacifici and H. Holzer (1976). Differences among sulfated proteoglycans synthesized in nonchondrogenic cells, presumptive chondroblasts, and chondroblasts. Proc. Natl. Acad. Sci., U.S.A. 73, 3224-3228.

Overman, D. O., R. E. Seegmiller and M. N. Runner (1972). Coenzyme competition and precursor specificity during teratogenesis induced by 6-aminonicotinamide. Dev. Biol. 28, 573-582.

Ritter, E. J., W. J. Scott and J. G. Wilson (1975). Inhibition of ATP synthesis associated with 6-aminonicotinamide (6-AN) teratogenesis in rat embryos. Teratology 12, 233-238.

Schubert, D. and M. Lacorbiere (1976). Phenotipic transformation of clonal myogenic cells to cells resembling chondrocytes. Proc. Natl. Acad. Sci. U.S.A. 73, 1989-1993.
Seegmiller, R. E., D. O. Overman and M. N. (1972). Histological and fine structure changes during chondrogenesis in micromelia induced by 6-aminonicotinamide. Dev. Biol. 28, 555-572.

Seegmiller, R. E. (1977). Time of onset and selective response of chondrogenic core of 5day chick limb after treatment with 6-aminonicotinamide. Dev. Biol. 58, 164-173.

Seegmiller, R. E., A. Horwitz and A. Dorfman (1980). Alterations of chondroitin sulfate synthesized by chick embryo cartilage cultured in the presence of 6-aminonicotinamide. $J$. Embryol. Exp. Morph. 59, 207-216.

Sheffield, V. C. and R. E. Seegmiller (1980). Impaired energy metabolism as an initial step in the mechanism for 6-aminonicotinamideinduced limb malformation. J. Embryol. Exp. Morph. 59, 217-222.

Suzuki, F., T. Yoneda and Y. Shimoyama (1976). Calcitonin and parathyroid hormone stimulation of acid mucopolysaccharide synthesis in cultured chondrocytes isolated from growth cartilage. FEBS Let. 70, 155-158.

Verrusio, A. C., D. R. Pollard and F. C. Fraser (1968). A cytoplasmically transmitted, dietdependent difference in response to the teratogenic effects of 6-aminonicotinamide. Science 160, 206-207. 